\title{
Unified Registration Model for Both Stationary and Mobile 3D Radar Alignment
}

\author{
Lei Chen, ${ }^{1}$ G. H. Wang, ${ }^{1}$ and Ilir F. Progri ${ }^{2}$ \\ ${ }^{1}$ Naval Aeronautical and Astronautical University, Yantai, Shandong 264001, China \\ ${ }^{2}$ Giftet Inc., 118 Heywood Street, No.3, Worcester, MA 01604, USA \\ Correspondence should be addressed to Lei Chen; chenlei_hjhy@yahoo.com
}

Received 16 July 2014; Revised 29 September 2014; Accepted 3 October 2014; Published 12 November 2014

Academic Editor: Peter Jung

Copyright (c) 2014 Lei Chen et al. This is an open access article distributed under the Creative Commons Attribution License, which permits unrestricted use, distribution, and reproduction in any medium, provided the original work is properly cited.

\begin{abstract}
For mobile radar, offset biases and attitude biases influence radar measurements simultaneously. Attitude biases generated from the errors of the inertial navigation system (INS) of the platform can be converted into equivalent radar measurement errors by three analytical expressions (range, azimuth, and elevation, resp.). These expressions are unique and embody the dependences between the offset and attitude biases. The dependences indicate that all the attitude biases can be viewed as and merged into some kind of offset biases. Based on this, a unified registration model (URM) is proposed which only contains radar "offset biases" in the form of system variables in the registration equations, where, in fact, the "offset biases" contain the influences of the attitude biases. URM has the same form as the registration model of stationary radar network where no attitude biases exist. URM can compensate radar offset and attitude biases simultaneously and has minor computation burden compared with other registration models for mobile radar network.
\end{abstract}

\section{Introduction}

With the increasing demands of the navigation accuracy in military and civilian applications, it is vitally important to fuse all the information from different sensors to obtain accurate target location estimate and comprehensive attribute information. However, before the benefits of multisensor integration can be realized, the sensor registration problem (or alignment) must be addressed. Sensor registration refers to the process of estimating sensor systematic biases (SBs) and compensating sensor measurements. If no registration is made, when raw measurements of different sensors are sent to the fusion center and transformed to a common reference frame, two kinds of adverse consequences would occur besides inaccurate location estimation: (1) data disassociation; (2) redundant tracks. In order to overcome the adverse influences mentioned above, a lot of registration methods were developed [1] based on stationary radar networks, and SBs in consideration only include range, gain of range, azimuth, and elevation biases referred to as offset biases (OBs).
Different from stationary radars, mobile radars are fixed on mobile carriers, in terms of installation methods, there are two kinds of platforms to fix radar: (1) gyrostabilized platforms (GSP) which can steadily follow systems referenced on local ENU (East-North-Up) frame [2]; (2) "unstabilized" GSP, namely, UGSP, directly fixed on platform and rotates with the platform simultaneously. GSP radar directly provides target coordinates (TCs) in the ENU reference frame. UGSP radar measurements are made in the measurement frame which should be converted to ENU frame by using attitude angles given by INS [3]. The common characteristic for both kinds of mobile radars is that they all need real-time attitude angle information to rectify radar sensitive axes influenced due to platform rotation. However, these attitude angles are biased because the accumulated errors in the inertial measurement units (IMU) of INS are referred to as attitude biases (ABs). Typically, when formulating 3D radar registration equations, both $\mathrm{OBs}$ and $\mathrm{ABs}$ are simultaneously selected as the state variables. If the system is unobservable (US), some of the biases cannot be estimated. Since both kinds of biases have the same influences on radar measurements (RMs), their 
dependencies should be analyzed first. If both kinds of biases are dependent, they should be combined to form new variables to make the system observable.

The concept of observability analysis (OA) first introduced for mobile radar registration (MRR) by Bar-Shalom [4] is not appropriate because the criterion he used is applied for linear time invariant system; however, the practical system is time varying. Then Herman and Poore [5] and Kragel et al. [6] gave registration model by using the least squares method and used the singular value decomposition (SVD) of the coefficient matrix $(\mathrm{CM})$ of the registration equations. Possible dependencies among all biases are analyzed qualitatively according to magnitudes of different column vector elements in the unitary matrix obtained from SVD. These criteria use the singularity of matrix to identify the observability of the system (OoS) and US easily in an optimal situation because when dependent variables are contained in the system, CM of the registration equations is singular. However, in practice, especially in noisy circumstances, CM is usually nonsingular even if variables are linearly dependent because small noises in radar measurements can easily make the singular CM nonsingular. In this situation, CM is obviously ill-conditioned and the misjudgement will result in poor Kalman filter (KF) estimation results [7]. So, in noisy circumstances or variables with slowly varying linear dependent coefficients in the registration equations, this SVD criterion can only give the qualitative instead of the exact dependent relations among different variables. Besides the methods above, the optimized bias estimation model (OBEM) was proposed by Wang et al. [8], where it was verified that when the azimuth and yaw biases are combined as one variable, the registration model is observable. The attitude bias conversion model (ABCM) [7] is proposed to explicitly establish nonlinear registration equations using linear dependencies among all biases. Both models can be improved with deeper understanding of the relationships between OBs and ABs.

The linearization-caused estimate errors were analyzed in [7], where it was proved that these errors are minor and can be omitted for MRR model. So, in this paper, the linearized registration model and $\mathrm{KF}$ are still used as the basic method to obtain the bias estimates. From the control theory perspective of view, for nonlinear control system with zero input control items, the linearized model can be used directly to construct observability matrix $(\mathrm{OM})$ to analyze the OoS [9]. Furthermore, Wang et al. [8] converted the $\mathrm{ABs}$ of the platform into radar measurement errors (MEs) by three analytical expressions. These expressions contain the TCs as well as the ABs as variables. Since the variations of the TCs between two consecutive observation instances are small compared with TCs, each expression can be divided into invariant and variant parts, respectively. The invariant part which is the majority in magnitude represents the dependencies between ABs and OBs, which can be proved by $\mathrm{OM}$ criterion. The variant part which is the minority in magnitude can be viewed as noise. These expressions are also deterministic and unique, which manifest that the dependencies between $\mathrm{ABs}$ and $\mathrm{OBs}$ are unavoidable and the MRR models are always US. Based on the analyses above we know that if the invariant parts converted from the $A B s$ merge into radar OBs, the system will be observable. At the same time, if the minor variant parts are viewed as noises, then all the ABs will be absent in the MRR model, and this model has the same form as that of the stationary radar network. So we call this model the unified registration model (URM) because it can unify the stationary and mobile radar network registration model into one frame. It should be noted that the only difference when URM is used for stationary and mobile radar registration is that for the latter the process noise variances will be enlarged because of the additional noises caused by the variant parts. Compared with other MRR models, URM proposed in this paper has the least number of variables (only has "OBs" as variables) and minor computation burden. The estimates of the "OBs" in fact contain the estimates of $\mathrm{ABs}$, and they can be used directly as OBs to compensate radar raw measurements. Usually the estimates of equivalent range and azimuth biases are accurate. Though many merits of URM, the equivalent elevation bias estimates are poor and need further improvement.

In order the simplify modeling, analysis, and assessment, other sources of biases in radar network such as sensor location bias errors and the timing biases are not discussed. It is assumed that both radars have accurate position information of themselves, and they are synchronized within the same sampling intervals.

Organization. In Section 2, a basic mathematical model for MRR is discussed. Observability analyses based on the basic model are described in Section 3. In Section 4, the detailed URM description based on observability analyses is presented and the variable attitude bias situation is discussed in Section 5. Simulation results with Cramer-Rao lower bounds (CRLBs) are given in Section 6. Finally, in Section 7, the final remarks of this study are given.

\section{Basic Registration Model}

Consider the $i$ th radar, where $i=\{1,2\}$, which is installed on the $i$ th moving carrier through three-axis gyrostabilized platform. The geographic coordinates of the $i$ th platform are latitude $L s_{i}$, longitude $R s_{i}$, and altitude $H_{i}$, which are known in real-time. Figure 1 provides an illustration of satelliteborne radar [10], where the gyrostabilized platform of the satellite can steadily track local ENU frame through three sets of IMUs and servo motors in east, north, and up directions, respectively. After initial alignment, when the attitude of the satellite changes, IMUs of each axis can sense this change and convert it to electric signal to drive the corresponding servo motor to rotate in the opposite directions; then the platform frame can catch up with the ENU frame in real-time.

However, for bias errors in IMUs, rectified axes of the gyrostabilized platform have a set of Eulerian angles with corresponding axes of ENU frame. These angles are ABs. Since radar is fixed on the platform, the sensitive axes of radar will rotate with the platform in ENU frame, and ABs of the platform can be passed on to RMs; these ABs together with radar OBs make radar measurement deviate from its true coordinates. 


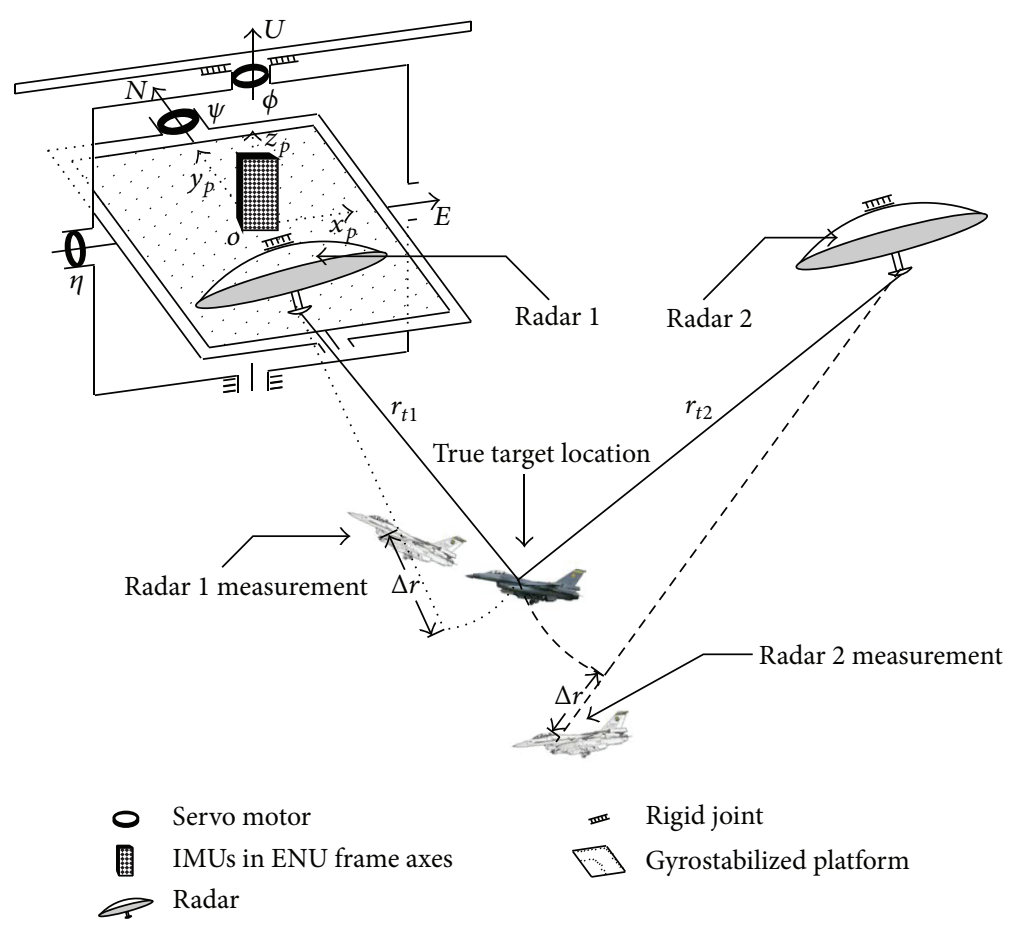

Figure 1: The diagram of mobile radar installed on the gyrostabilized platform.

Usually, ABs are defined as yaw bias $\Delta \phi$, pitch bias $\Delta \eta$, and roll bias $\Delta \psi$, respectively [11], which can be seen in Figure 2(a). For ENU frame, its origin $o$ is located at the center of the gyrostabilized platform; three mutually orthogonal axes $x, y$, and $z$ refer to the directions of east, north, and up, respectively. The plane xoy is horizontal. We define the output Cartesian coordinates of the gyrostabilized platform as the platform frame. The platform frame has the same origin with ENU; Figure 2(a) shows the conversion process from the platform frame to ENU, where $x_{p}, y_{p}$, and $z_{p}$ denote $x, y$, and $z$ axes of the platform frame, respectively, and the axes drawn in dashed lines are the intermediate axes. As shown in Figure 2(a), the transformation of TCs from the platform frame to ENU is accomplished by first rotating about the $y$-axis of the platform frame by the roll angle $\Delta \psi$, then rotating about the intermediate $x$-axis by the pitch angle $\Delta \eta$, and rotating about the final $z$-axis by the yaw angle $\Delta \phi$. Customarily, the polarity definitions of $\Delta \phi$ and $\Delta \psi$ abide by the left-hand rule, and $\Delta \eta$ abides by the right-hand rule.

Figure 2(b) depicts radar measurement in the platform frame, where radar $i$ is located at the origin $o$ and $T_{t}$ denotes the true target location. $T_{m}^{\prime}$ denotes the ghost target influenced by the azimuth bias only and $T_{m}$ denotes the ghost target influenced by all the biases. $o_{p}, o_{m}^{\prime}$, and $o_{m}$ denote the corresponding projections on the platform frame $x_{p} o y_{p}$ plane, respectively. $o_{e}$ denotes the projection of the true target on the horizontal plane of the ENU frame. The measurements of target from the $i$ th radar include the range $r_{i}$, azimuth $\theta_{i}$ (the true north corresponds to $\theta=0$, and the clockwise direction denotes the increment of $\theta$ ), and elevation $\varepsilon_{i}$, which contain the true target position information (such as the true range $r_{i t}$, azimuth $\theta_{i t}$, and elevation $\varepsilon_{i t}$ ), radar OBs (such as the range bias $\Delta r_{i}$, the gain of range $k_{r i}$ which arise from (or in) atmospheric refraction [12], azimuth $\Delta \theta_{i}$, and elevation $\Delta \varepsilon_{i}$. Polarity definitions of $\Delta \theta_{i}$ and $\Delta \varepsilon_{i}$ are identical to $\theta$ and $\varepsilon$, resp.), $A B s$ of the gyrostabilized platform, and random MEs (such as the range error $\delta_{r i}$, azimuth $\delta_{\theta i}$, and elevation $\delta_{\varepsilon i}$ ). Random MEs are zero-mean Gaussian with known standard deviations. Figure 2(b) also shows that since the existence of $\mathrm{ABs}$, the true target azimuth and elevation in platform frame are different from those in the ENU frame.

The main objective for MRR is to simultaneously estimate $\mathrm{OBs}$ and $\mathrm{ABs}$ using both radars raw measurements. As shown in Figure 3, the mathematical registration models are established through the following process: (1) radar OBs, random MEs, and equivalent MEs caused by ABs included in the raw measurements are removed to obtain the true target coordinates (TTCs) in the ENU frame; (2) the conversion from ENU to ECEF frame is used to obtain TTCs in the common reference frame; (3) the registration equations can be established according to the fact that TTCs obtained from two different RMs are equal when they are converted to the common reference frame; (4) bias estimations (BEs) can be obtained by using different estimation algorithms. Initially all $\mathrm{OBs}$ and $\mathrm{ABs}$ are assumed constants and later as varying biases. The following are the main procedures.

Let the column vectors

$$
\begin{aligned}
& \mathbf{X}_{i-p}(k)=\left[x_{i-p}(k), y_{i-p}(k), z_{i-p}(k)\right]^{T}, \\
& \mathbf{X}_{i \mathrm{ENU}}(k)=\left[x_{i-\mathrm{ENU}}(k), y_{i-\mathrm{ENU}}(k), z_{i \mathrm{ENU}}(k)\right]^{T}, \\
& \mathbf{X}_{i_{-} \mathrm{ECEF}}(k)=\left[x_{i_{\text {ECEF }}}(k), y_{i_{-} \mathrm{ECEF}}(k), z_{i_{-} \mathrm{ECEF}}(k)\right]^{T}
\end{aligned}
$$




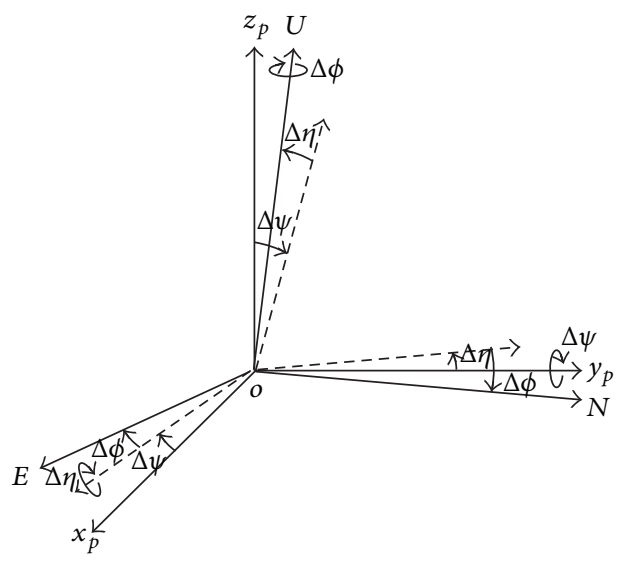

(a)

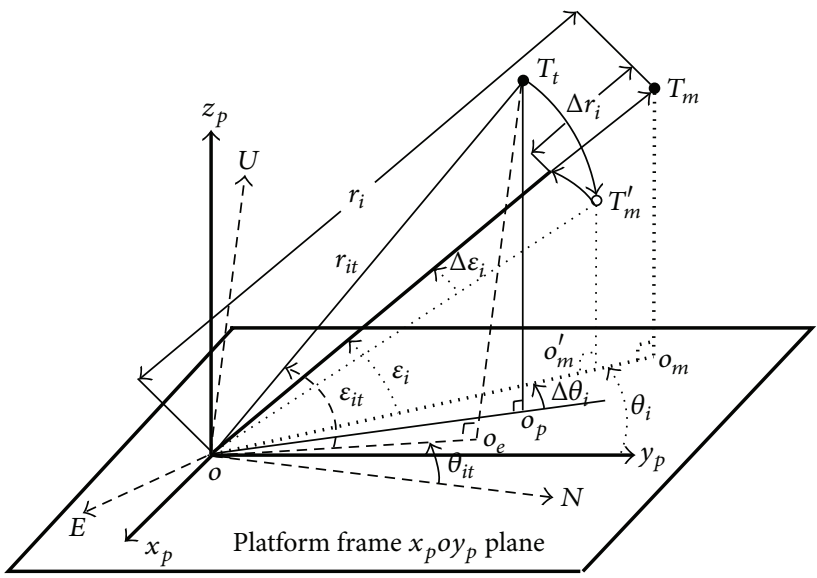

(b)

FIgURE 2: (a) Conversion from the platform frame to ENU and (b) measurement in platform frame.

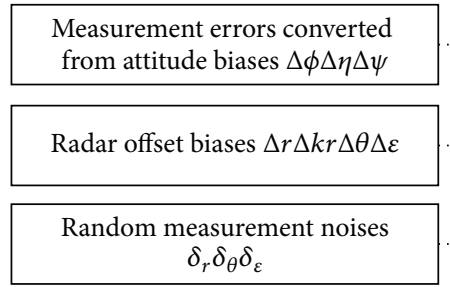
$\delta_{r} \delta_{\theta} \delta_{\varepsilon}$
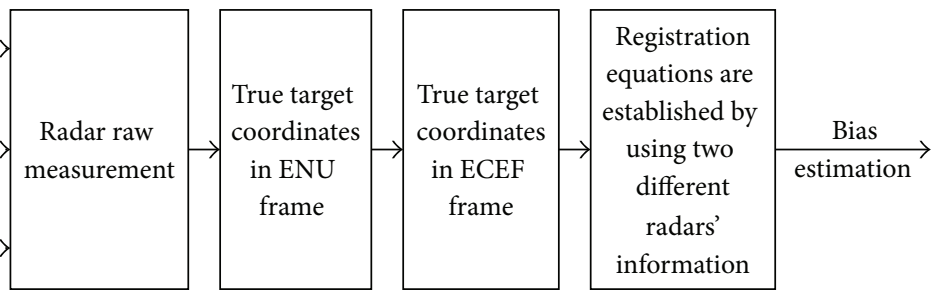

Figure 3: The flow chart for MRR. represent the true rectangular coordinates of the target at the observation instance $k$ in the platform frame, ENU frame, and ECEF frame, respectively [13], of the $i$ th sensor. The superscript " $T$ " denotes matrix or vector transposition. These column vectors are also nonlinear vector functions. Sensor measurements, OBs, $\mathrm{ABs}$, and random MEs are included in each element of the vectors.

Usually, all OBs and random MEs are small in magnitudes, in order to simplify the development of registration equations below; $\mathbf{X}_{i_{-} p}(k)$ can be approximated by first-order Maclaurin series about OBs and random MEs as

$$
\mathbf{X}_{i-p}(k) \approx \mathbf{X}_{i}(k)+\mathbf{A}_{i}(k) \boldsymbol{\beta}_{i}(k)+\mathbf{C}_{i}(k) \mathbf{w}_{i}(k),
$$

where

$$
\begin{aligned}
& \boldsymbol{\beta}_{i}(k)=\left[\Delta r_{i}, k_{r i}, \Delta \theta_{i}, \Delta \varepsilon_{i}\right]^{T}, \\
& \mathbf{w}_{i}(k)=\left[\delta_{r i}(k), \delta_{\theta i}(k), \delta_{\varepsilon i}(k)\right]^{T}, \\
& \mathbf{X}_{i}(k)=\left[x_{i}(k), y_{i}(k), z_{i}(k)\right]^{T}=\left.\mathbf{X}_{i-p}(k)\right|_{\substack{\boldsymbol{\beta}_{i}(k)=\mathbf{0} \\
\mathbf{w}_{i}(k)=\mathbf{0}}}, \\
& \mathbf{A}_{i}(k)=\left.\frac{\partial \mathbf{X}_{i-p}(k)}{\partial \boldsymbol{\beta}_{i}(k)}\right|_{\substack{\beta_{\mathbf{w}_{i}}(k)=\mathbf{0} \\
\mathbf{w}_{i}(k)=\mathbf{0}}}, \\
& \mathbf{C}_{i}(k)=\left.\frac{\partial \mathbf{X}_{i-p}(k)}{\partial \mathbf{w}_{i}(k)}\right|_{\substack{\boldsymbol{\beta}_{\mathbf{i}}(k)=\mathbf{0} \\
\mathbf{w}_{i}(k)=\mathbf{0}}} .
\end{aligned}
$$

According to the polarity definition of $\mathrm{ABs}$ and the rotation transformation order, the transition from the platform frame to ENU is given by [7]

$$
\mathbf{X}_{i \text { ENU }}(k)=\mathbf{T}_{i_{-} \text {2 } \mathrm{ENU}}(k) \mathbf{X}_{i_{-} p}(k),
$$

where $\mathbf{T}_{i_{-} \mathrm{p} 2 \mathrm{ENU}}$ denotes the rotation matrix from the platform frame to ENU, which is an orthogonal matrix; that is, $T_{i_{-} \text {p2ENU }}^{-1}=T_{i_{-} \text {2 } 2 \mathrm{ENU}}^{T}=T_{i_{\text {ENU } 2 p}}$, and [8]

$$
\mathbf{T}_{i \_p 2 \mathrm{ENU}}=\left[\begin{array}{lll}
t_{11 \_i} & t_{12 i} & t_{13 i} \\
t_{21 \_i} & t_{22 i} & t_{23 i i} \\
t_{31 \_i} & t_{32 i} & t_{33 i}
\end{array}\right],
$$

$$
t_{11_{i} i}=\cos \left(\Delta \psi_{i}\right) \cos \left(\Delta \phi_{i}\right)-\sin \left(\Delta \psi_{i}\right) \sin \left(\Delta \eta_{i}\right) \sin \left(\Delta \phi_{i}\right) \text {, }
$$

$$
t_{12_{-} i}=\cos \left(\Delta \eta_{i}\right) \sin \left(\Delta \phi_{i}\right),
$$

$t_{13 \_i}=-\sin \left(\Delta \psi_{i}\right) \cos \left(\Delta \phi_{i}\right)-\cos \left(\Delta \psi_{i}\right) \sin \left(\Delta \eta_{i}\right) \sin \left(\Delta \phi_{i}\right)$,

$$
\begin{gathered}
t_{21 \_i}=-\cos \left(\Delta \psi_{i}\right) \sin \left(\Delta \phi_{i}\right)-\sin \left(\Delta \psi_{i}\right) \sin \left(\Delta \eta_{i}\right) \cos \left(\Delta \phi_{i}\right), \\
t_{22_{i} i}=-\cos \left(\Delta \eta_{i}\right) \cos \left(\Delta \phi_{i}\right), \\
t_{23 \_i}=\sin \left(\Delta \psi_{i}\right) \sin \left(\Delta \phi_{i}\right)-\cos \left(\Delta \psi_{i}\right) \sin \left(\Delta \eta_{i}\right) \cos \left(\Delta \phi_{i}\right),
\end{gathered}
$$




$$
\begin{gathered}
t_{31 i}=\sin \left(\Delta \psi_{i}\right) \cos \left(\Delta \eta_{i}\right), \\
t_{32 i}=\sin \left(\Delta \eta_{i}\right), \\
t_{33 i}=\cos \left(\Delta \psi_{i}\right) \cos \left(\Delta \eta_{i}\right) .
\end{gathered}
$$

Similarly, (5a) can be approximated by the first-order Maclaurin series about ABs as

$$
\mathbf{T}_{i_{-} \text {p2ENU }} \approx \mathbf{I}+\Delta_{i}(k)
$$

where $\mathbf{I}$ is a $3 \times 3$ identity matrix, and

$$
\Delta_{i}(k)=\left[\begin{array}{ccc}
0 & \Delta \phi_{i} & -\Delta \psi_{i} \\
-\Delta \phi_{i} & 0 & -\Delta \eta_{i} \\
\Delta \psi_{i} & \Delta \eta_{i} & 0
\end{array}\right]
$$

Substituting (2) and (6) into (4) and omitting the higher order terms, (4) can be expanded as

$$
\begin{aligned}
& \mathbf{X}_{i \_\mathrm{ENU}}(k) \\
& \approx \underbrace{\left[\mathbf{I}+\boldsymbol{\Delta}_{i}(k)\right]\left[\mathbf{X}_{i}(k)+\mathbf{A}_{i}(k) \boldsymbol{\beta}_{i}(k)+\mathbf{C}_{i}(k) \mathbf{w}_{i}(k)\right]}_{\approx \mathbf{X}_{i}(k)+\mathbf{A}_{i}(k) \boldsymbol{\beta}_{i}(k)+\mathbf{C}_{i}(k) \mathbf{w}_{i}(k)+\boldsymbol{\Delta}_{i}(k) \mathbf{X}_{i}(k)}, \\
& \mathbf{X}_{i \_\mathrm{ENU}}(k)+\left[-\mathbf{A}_{i}(k) \boldsymbol{\beta}_{i}(k)-\mathbf{C}_{i}(k) \mathbf{w}_{i}(k)-\boldsymbol{\Delta}_{i}(k) \mathbf{X}_{i}(k)\right] \\
& \approx \mathbf{X}_{i}(k) .
\end{aligned}
$$

Comparing (8a) and (8b) with (2), it is obvious that target location errors caused by ABs can be expressed by the last term of the left hand side of (8b). Omitting the subscript " $i$ " and time stamp " $k$," the errors can be written as

$$
\left[\begin{array}{l}
e_{x} \\
e_{y} \\
e_{z}
\end{array}\right]=-\Delta \mathbf{X}=-\left[\begin{array}{ccc}
0 & \Delta \phi & -\Delta \psi \\
-\Delta \phi & 0 & -\Delta \eta \\
\Delta \psi & \Delta \eta & 0
\end{array}\right]\left[\begin{array}{l}
x \\
y \\
z
\end{array}\right],
$$

where $e_{x}, e_{y}$, and $e_{z}$ denote the errors caused by ABs in $x, y$, and $z$ coordinates, respectively.

Then, the equivalent range MEs caused by $\mathrm{ABs}$ can be expressed as [8]

$$
\begin{aligned}
\Delta r_{c}(\Delta \phi, \Delta \eta, \Delta \psi)= & \sqrt{\left(x+e_{x}\right)^{2}+\left(y+e_{y}\right)^{2}+\left(z+e_{z}\right)^{2}} \\
& -\sqrt{x^{2}+y^{2}+z^{2}} .
\end{aligned}
$$

Substituting (9) into (10) and selecting ABs in (10) as variables, the first-order Maclaurin series expansion of (10) can be written as

$$
\Delta r_{c}(\Delta \phi, \Delta \eta, \Delta \psi)=0+o(\Delta \phi, \Delta \eta, \Delta \psi)
$$

Similarly, the equivalent azimuth measurement error is

$$
\Delta \theta_{c}=-\Delta \phi+\frac{y z \Delta \psi-x z \Delta \eta}{x^{2}+y^{2}}+o(\Delta \phi, \Delta \eta, \Delta \psi) .
$$

The equivalent elevation measurement error is

$$
\Delta \varepsilon_{c}=\frac{-x \Delta \psi-y \Delta \eta}{\sqrt{x^{2}+y^{2}}}+o(\Delta \phi, \Delta \eta, \Delta \psi) .
$$

Since errors caused by ABs are equivalent to MEs, then TTCs in ENU frame can be written out directly without using rotation transformation as

$$
\begin{gathered}
\mathbf{X}_{i_{-} \mathrm{ENU}}(k)=\gamma_{r_{i}}(k) \times\left\{\begin{array}{c}
\sin \left[\gamma_{\theta i}(k)\right] \cos \left[\gamma_{\varepsilon i}(k)\right] \\
\cos \left[\gamma_{\theta i}(k)\right] \cos \left[\gamma_{\varepsilon i}(k)\right] \\
\sin \left[\gamma_{\varepsilon i}(k)\right]
\end{array}\right\}, \\
\gamma_{r_{i}}(k)=r_{i}(k)-\Delta r_{i}-k_{r i} r_{i t}(k)-\Delta r_{c i}(k)-\delta_{r i}(k), \\
\gamma_{\theta i}(k)=\theta_{i}(k)-\Delta \theta_{i}-\Delta \theta_{c i}(k)-\delta_{\theta i}(k), \\
\gamma_{\varepsilon i}(k)=\varepsilon_{i}(k)-\Delta \varepsilon_{i}-\Delta \varepsilon_{c i}(k)-\delta_{\varepsilon i}(k) .
\end{gathered}
$$

Substituting (11)-(13) into (14a) and omitting time stamp " $k$ ” for brevity, (14a) produces

$$
\begin{gathered}
\gamma_{r_{i}}=r_{i}-\Delta r_{i}-k_{r i} r_{i t}-\delta_{r i}, \\
\gamma_{\theta i}=\theta_{i}-\Delta \theta_{i}+\Delta \phi_{i}-\frac{y_{i} z_{i} \Delta \psi_{i}-x_{i} z_{i} \Delta \eta_{i}}{x_{i}^{2}+y_{i}^{2}}-\delta_{\theta i}, \\
\gamma_{\varepsilon i}=\varepsilon_{i}-\Delta \varepsilon_{i}+\frac{x_{i} \Delta \psi_{i}+y_{i} \Delta \eta_{i}}{\sqrt{x_{i}^{2}+y_{i}^{2}}}-\delta_{\varepsilon i} .
\end{gathered}
$$

The transition of TTCs from ENU to ECEF frame is given by Zhou et al. [14] as

$$
\mathbf{X}_{i \text { ECEF }}(k)=\mathbf{X}_{i s}(k)+\mathbf{T}_{i}(k) \times \mathbf{X}_{i \_\mathrm{ENU}}(k),
$$

where $\mathbf{X}_{i s}$ denotes the $i$ th radar ECEF coordinates converted from its geographic coordinates, and $\mathbf{T}_{i}$ is the rotation matrix. Both variables are only correlated with radar geographic coordinates at time $k$.

Since

$$
\mathbf{X}_{1 \_ \text {ECEF }}(k)=\mathbf{X}_{2 \_ \text {ECEF }}(k) \text {. }
$$

substituting (14a) and (16) into (17), the first-order linearized registration model can be approximated in the form of

$$
\mathbf{Z}(k)=\mathbf{H}(k) \boldsymbol{\beta}(k)+\mathbf{C}(k) \mathbf{w}(k),
$$

where $\boldsymbol{\beta}^{T}=\left[\boldsymbol{\beta}_{1}^{T}, \boldsymbol{\beta}_{2}^{T}\right]$ denotes the state vector consisting of all OBs and ABs of both radars. $\mathbf{w}^{T}=\left[\mathbf{w}_{1}^{T}, \mathbf{w}_{2}^{T}\right]$ is a column vector which contains both radars' measurement noises.

\section{Observability Analysis}

Assuming a constant bias registration model, for which the state equations can be described as

$$
\boldsymbol{\beta}(k+1)=\mathbf{I} \boldsymbol{\beta}(k),
$$

I denotes the corresponding unit matrix. 
First-order linearized equivalent measurement equations are assumed to have the similar form of (18).

According to dynamic equations (18) and (19), the $N$-step random OM can be written as [8]

$$
\mathbf{M}(k, k-N+1)=\sum_{i=k-N+1}^{k} \underbrace{\mathbf{H}^{T}(i) \mathbf{C}(i) \mathbf{R}^{-1}(i) \mathbf{C}^{T}(i) \mathbf{H},}_{\mathbf{H}^{T}(i) \mathbf{C}(i) \mathbf{R}^{-1}(i) \mathbf{C}^{T}(i) \mathbf{H}(i)}
$$

where $E\left\{\mathbf{w}(k) \mathbf{w}^{T}(k)\right\}=\mathbf{R}(k)$ and $E\{x\}$ denotes the expectation of " $x$." $N$ is a positive integer which is unrelated to $k$. $\mathbf{R}(i)$ in (20) is a constant matrix at different time because the RNs have the same variances at different time.

The system is observable (OS) when OM is positive definite [15]. In the following three cases, the observability of the different system is analyzed, respectively.

Condition 1. When $\mathbf{C}(i)$ is a constant matrix then two of the column vectors in the coefficient matrix $\mathbf{H}(i)$ are proportional-US.

Proof. Without loss of generality, assuming that column vectors $\mathbf{h}_{1}(i)$ and $\mathbf{h}_{2}(i)(i=k-N+1, \ldots, k)$ are first and second column vectors in $\mathbf{H}(i)$, respectively, and satisfy $\mathbf{h}_{1}(i) / \mathbf{h}_{2}(i)=a$, where the proportionality factor $a$ is a constant, then OM can be written as

$$
\mathbf{M}(k, k-N+1)=\sum_{i=k-N+1}^{k} \mathbf{H}^{T}(i) \mathbf{C R}^{-1} \mathbf{C}^{T} \mathbf{H}(i) .
$$

Assuming that $\mathbf{m}(i)=\mathbf{H}^{T}(i) \mathbf{C R}^{-1} \mathbf{C}^{T} \mathbf{H}(i)$ and $\mathbf{v}_{1}(i)$ and $\mathbf{v}_{2}(i)$ are the first and second row vectors in $\mathbf{m}(i)$, respectively, then it is obvious that $\mathbf{v}_{1}(i) / \mathbf{v}_{2}(i)=a$ as follows:

$$
\frac{\mathbf{v}_{1}(k-N+1)+\mathbf{v}_{1}(k-N+2)+\cdots+\mathbf{v}_{1}(k)}{\mathbf{v}_{2}(k-N+1)+\mathbf{v}_{2}(k-N+2)+\cdots+\mathbf{v}_{2}(k)}=a ;
$$

that is, the first and second row vectors of OM $\mathbf{M}$ are proportional and the proportionality factor is $a$. Then $\mathbf{M}=$ 0 -US.

Condition 2. When $\mathbf{C}(i)$ or $\mathbf{H}(i)$ is time-varying then the system is observable or-OS.

Proof. In this case, $\mathbf{m}(i)=\mathbf{H}^{T}(i) \mathbf{C}(i) \mathbf{R}^{-1} \mathbf{C}^{T}(i) \mathbf{H}(i)$ is an $n \times n$ matrix, and the rank of $\mathbf{m}(i)$ is 3 because the rank of $\mathbf{H}(i)$ is 3 . Since $\mathbf{C}(i)$ or $\mathbf{H}(i)$ is different in each time, when

$$
N \geq\left[\frac{n}{3}\right]
$$

where the symbol $[x]$ denotes the nearest integer greater than or equal to $x$, the rank of $\sum_{i=k-N+1}^{k} \mathbf{m}(i)$ is equal to $N$, and OM $\mathbf{M}$ is positive definite-OS.

Condition 3. When $\mathbf{C}(i)$ and the proportionality factor of two column vectors in $\mathbf{H}(i)$ vary slowly between different observation time, though OS, they can be tackled as US.
According to the proof of Condition 2, though the system is observable, the condition number of the coefficient matrix $\mathbf{H}(i)$ is very big because two column vectors in $\mathbf{H}(i)$ are proportional, and solutions of KF for the system are sensitive to noises. Since KF is an iterative algorithm, in each computational cycle, the variables in the state vector corresponding to both proportional column vectors in $\mathbf{H}(i)$ should be combined as one variable; then the system is observable, and the dimension of the state vector can also be decreased. Otherwise, if they are estimated separately, the solutions for each variable are unbelievable. As for variations between two different observation instances, if the magnitudes of the variation are small, they can be viewed as noises. Based on this, (19) can be modified as

$$
\boldsymbol{\beta}^{\prime}(k+1)=\boldsymbol{\beta}^{\prime}(k)+\mathbf{w}_{\boldsymbol{\beta}^{\prime}}(k+1),
$$

where $\boldsymbol{\beta}^{\prime}$ denotes the combined vector and $\mathbf{w}_{\boldsymbol{\beta}^{\prime}}$ denotes the remaining minor noises caused by the independent parts.

\section{Unified Registration Model}

Since variations of TCs are usually small compared with their coordinates, in MRR (18) derived from (15a), (15b), and (15c), coefficients of $\mathrm{ABs}$ and $\mathrm{OBs}$ can be divided into two parts: the invariant (or proportional) and variant parts which satisfy Conditions 1 and 2, respectively. According to analyses made for Condition 3, it is optimum to select the united biases as state vector as

$$
\boldsymbol{\beta}_{i}^{\prime}(k)=\left[\Delta r_{i}, k_{r i}, \Delta \theta_{i}^{\prime}(k), \Delta \varepsilon_{i}^{\prime}(k)\right]^{T},
$$

where

$$
\begin{gathered}
\Delta \theta_{i}^{\prime}(k)=\Delta \theta_{i}-\Delta \phi_{i}+\frac{y_{i}(k) z_{i}(k) \Delta \psi_{i}-x_{i}(k) z_{i}(k) \Delta \eta_{i}}{x_{i}^{2}(k)+y_{i}^{2}(k)} \\
\Delta \varepsilon_{i}^{\prime}(k)=\Delta \varepsilon_{i}-\frac{x_{i}(k) \Delta \psi_{i}+y_{i}(k) \Delta \eta_{i}}{\sqrt{x_{i}^{2}(k)+y_{i}^{2}(k)}}
\end{gathered}
$$

According to above analyses, in (25) it is optimum to select

$$
\Delta r_{i}^{\prime}(k)=\Delta r_{i}+k_{r i} r_{i t}(k) ;
$$

however, it is not the best although it decreases the dimension of the state vector. The reason is that the constant models are assumed for $\mathrm{OBs}$ and $\mathrm{ABs}$, and coupling influences on the radar range measurement caused by $\mathrm{ABs}$ are zeros (which can be seen from (11)). The gross range bias only correlates with the target range $\left(r_{i}\right)$ and is independent with TCs $\left(x_{i}, y_{i}, z_{i}\right)$. If the range bias uses (28) as the state variable, its state equation will be in the form of a constant plus a noise, which is inaccurate compared to the model using (25). Moreover, it will make the computation of the cross covariance of the state vectors more complicated and the estimation accuracies will be decreased (the simulation results made by authors have verified the results; for the limitation of the article length, the corresponding algorithm will not be given). 
Hence, it is preferable to estimate $\Delta r_{i}$ and $k_{r i}$ individually. However, the estimation results for both variables should be used simultaneously when rectifying radar raw measurements. It is meaningless to evaluate their estimation performance individually.

In the new state vector (25), variables $\Delta \theta_{i}^{\prime}$ and $\Delta \varepsilon_{i}^{\prime}$ are no longer constants; they are varying with different target locations. Equations (26) and (27) can be differentiated individually about the time argument as

$$
\begin{aligned}
d\left(\Delta \theta^{\prime}\right) & =d\left(\frac{y z \Delta \psi-x z \Delta \eta}{x^{2}+y^{2}}\right)=\frac{a_{x} d x+a_{y} d y+a_{z} d z}{\left(x^{2}+y^{2}\right)^{2}} \\
a_{x}= & -\left(x^{2}+y^{2}\right) z \Delta \eta-2 x(y z \Delta \psi-x z \Delta \eta) \\
a_{y}= & \left(x^{2}+y^{2}\right) z \Delta \psi-2 y(y z \Delta \psi-x z \Delta \eta) \\
& a_{z}=\left(x^{2}+y^{2}\right) y \Delta \psi-\left(x^{2}+y^{2}\right) x \Delta \eta
\end{aligned}
$$

The subscript " $i$ " which identifies different radars and time stamp " $k$ " are omitted in (29a) and (30) for brevity. Assume that velocities of the target in $x, y$, and $z$ directions in radar $i$ ENU frame are mutually independent zero-mean Gaussian white noises whose standard deviations are $\sigma_{v_{x}}, \sigma_{v_{y}}$, and $\sigma_{v_{z}}$, respectively. According to (29a) and (30), $d\left(\Delta \theta^{\prime}\right)$ and $d\left(\Delta \varepsilon^{\prime}\right)$ are zero-mean Gaussian random processes, whose covariance can be written out as

$$
\begin{aligned}
\sigma_{d\left(\Delta \theta^{\prime}\right)}^{2}= & \operatorname{Var}\left\{d\left(\Delta \theta^{\prime}\right)\right\} \\
= & \operatorname{Var}\left\{\frac{a_{x} T v_{x}+a_{y} T v_{y}+a_{z} T v_{z}}{\left(x^{2}+y^{2}\right)^{2}}\right\} \\
= & \frac{b_{x}^{2} T^{2} \sigma_{v_{x}}^{2}+b_{y}^{2} T^{2} \sigma_{v_{y}}^{2}+b_{z}^{2} T^{2} \sigma_{v_{z}}^{2}}{\left(x^{2}+y^{2}\right)^{4}}, \\
& b_{x}=\left(x^{2}-y^{2}\right) z \Delta \eta-2 x y z \Delta \psi, \\
& b_{y}=\left(x^{2}-y^{2}\right) z \Delta \psi+2 x y z \Delta \eta, \\
b_{z}= & \left(x^{2}+y^{2}\right) y \Delta \psi-\left(x^{2}+y^{2}\right) x \Delta \eta,
\end{aligned}
$$

$$
\begin{array}{r}
\sigma_{d\left(\Delta \varepsilon^{\prime}\right)=\operatorname{Var}\left\{d\left(\Delta \varepsilon^{\prime}\right)\right\}}^{2}=\operatorname{Var}\left\{c_{x} v_{x}+c_{y} v_{y}\right\} \\
=c_{x}^{2} \operatorname{Var}\left\{v_{x}\right\}+c_{y}^{2} \operatorname{Var}\left\{v_{y}\right\}=c_{x}^{2} \sigma_{v_{x}}^{2}+c_{y}^{2} \sigma_{v_{y}}^{2}, \\
c_{x}=\frac{\left(-y^{2} \Delta \psi+x y \Delta \eta\right) T}{\left(x^{2}+y^{2}\right)^{3 / 2}}, \\
c_{y}=\frac{\left(-x^{2} \Delta \eta+x y \Delta \psi\right) T}{\left(x^{2}+y^{2}\right)^{3 / 2}},
\end{array}
$$

where $T$ denotes radar scanning period. $\operatorname{Var}\{x\}$ denotes the variance of " $x$." The cross covariance can be written as

$$
\begin{array}{r}
\sigma_{d\left(\Delta \theta^{\prime}\right) d\left(\Delta \varepsilon^{\prime}\right)}^{2}=\sigma_{d\left(\Delta \varepsilon^{\prime}\right) d\left(\Delta \theta^{\prime}\right)}^{2}=E\left\{d\left(\Delta \theta^{\prime}\right) d\left(\Delta \varepsilon^{\prime}\right)\right\} \\
=E\left\{g_{x} v_{x}^{2}+g_{y} v_{y}^{2}\right\}=g_{x} \sigma_{v_{x}}^{2}+g_{y} \sigma_{v_{y}}^{2}, \\
g_{x}=\frac{b_{x}\left(-y^{2} \Delta \psi+x y \Delta \eta\right) T^{2}}{\left(x^{2}+y^{2}\right)^{7 / 2}}, \\
g_{y}=\frac{b_{y}\left(-x^{2} \Delta \eta+x y \Delta \psi\right) T^{2}}{\left(x^{2}+y^{2}\right)^{7 / 2}} .
\end{array}
$$

$E\{x\}$ denotes the expectation of " $x$." According to the state vector (25), substituting (14a) and (16) into (17) and using the first-order Maclaurin series expansion about OBs, ABs, and random measurement noises, the registration equations can be approximated as

$$
\begin{aligned}
\mathbf{Z}_{\mathrm{URM}}(k)= & {\left[\mathbf{T}_{1}(k) \mathbf{A}_{1}(k),-\mathbf{T}_{2}(k) \mathbf{A}_{2}(k)\right] \boldsymbol{\beta}_{\mathrm{URM}}(k) } \\
& +\left[\mathbf{T}_{1}(k) \mathbf{C}_{1}(k),-\mathbf{T}_{2}(k) \mathbf{C}_{2}(k)\right]\left[\begin{array}{l}
\mathbf{w}_{1}(k) \\
\mathbf{w}_{2}(k)
\end{array}\right],
\end{aligned}
$$

where $\mathbf{T}_{i}(k), \mathbf{A}_{i}(k), \mathbf{C}_{i}(k)$, and $\mathbf{w}_{i}(k)$ are completely the same as (2), and

$$
\begin{gathered}
\mathbf{Z}_{\mathrm{URM}}(k)=\mathbf{X}_{2 s}(k)-\mathbf{X}_{1 s}(k)+\mathbf{T}_{2}(k) \mathbf{X}_{2}(k)-\mathbf{T}_{1}(k) \mathbf{X}_{1}(k), \\
\boldsymbol{\beta}_{\mathrm{URM}}(k)=\left[\boldsymbol{\beta}_{1}^{\prime}(k)^{T}, \boldsymbol{\beta}_{2}^{\prime}(k)^{T}\right]^{T} .
\end{gathered}
$$

According to the analyses above, each systematic bias can be equivalent to a constant plus a zero-mean Gaussian white noise. The dynamic equation for them can be written as

$$
\boldsymbol{\beta}_{\mathrm{URM}}(k+1)=\boldsymbol{\beta}_{\mathrm{URM}}(k)+\mathbf{w}_{\boldsymbol{\beta}}(k+1),
$$

where

$$
\mathbf{w}_{\boldsymbol{\beta}}=\left[0,0, w_{d\left(\Delta \theta_{1}^{\prime}\right)}, w_{d\left(\Delta \varepsilon_{1}^{\prime}\right)}, 0,0, w_{d\left(\Delta \theta_{2}^{\prime}\right)}, w_{d\left(\Delta \varepsilon_{2}^{\prime}\right)}\right]^{T} .
$$

The covariance matrix of $\mathbf{w}_{\beta}$ can be written as

$$
\mathbf{Q}=\operatorname{diag}\left(\left[\mathbf{Q}_{\boldsymbol{\beta}_{1}^{\prime}}, \mathbf{Q}_{\boldsymbol{\beta}_{2}^{\prime}}\right]\right),
$$


where $\operatorname{diag}(\mathbf{x})$ denotes a matrix whose diagonal elements are composed of the vector $\mathbf{x}$ and the other elements of the matrix are zeros, and

$$
\begin{gathered}
\mathbf{Q}_{\beta_{i}^{\prime}}=\left[\begin{array}{cccc}
0 & 0 & 0 & 0 \\
0 & 0 & 0 & 0 \\
0 & 0 & q_{33} & q_{34} \\
0 & 0 & q_{43} & q_{44}
\end{array}\right], \\
q_{33}=\sigma_{d\left(\Delta \theta_{i}^{\prime}\right)}^{2}, \quad q_{34}=\sigma_{d\left(\Delta \theta_{i}^{\prime}\right) d\left(\Delta \varepsilon_{i}^{\prime}\right)}^{2}, \\
q_{43}=\sigma_{d\left(\Delta \varepsilon_{i}^{\prime}\right) d\left(\Delta \theta_{i}^{\prime}\right)}^{2}=q_{34}, \quad q_{44}=\sigma_{d\left(\Delta \varepsilon_{i}^{\prime}\right)}^{2} .
\end{gathered}
$$

The linearized registration model is composed of (34) and (36), which unites radar OBs and the equivalent radar MEs caused by $\mathrm{ABs}$ as one variable. The system is observable and the estimation results can compensate the influences of $\mathrm{OBs}$ and $\mathrm{ABs}$ simultaneously. It also has the form of stationary radar registration model which has no $\mathrm{ABs}$ to be considered, and it is the extension of the latter. So, this model is called URM. Figure 4 is the block diagram of URM which manifests that the only difference between mobile and stationary radar registration when they use URM is that the former uses additional Gaussian white noises as part of azimuth and elevation biases. This is also the difference between URM and OBEM [1] despite the fact that they use the same state vector and equivalent measurement equations.

In the computation of the covariance matrix of the state vector, the standard deviation of the target velocities and the true $\mathrm{ABs}$ should be given previously. However, their true values cannot be obtained in practice; then it is feasible to use possible maximum values to replace them, which will inevitably increase the estimation errors. In fact, from (31a)-(33) the variances caused by the variations of the target locations are very small in magnitude; therefore, these approximations work reasonably well in practice.

\section{Varying ABs Situation}

Before this section, all $\mathrm{OBs}$ and $\mathrm{ABs}$ were assumed to be constants. In this discussion they are extended as varying biases. Despite the fact that many models such as constant or one-order Gauss-Markov process have been used for biases [4], we propose the more general model for varying bias $\beta$ as

$$
\beta(k)=\beta_{0}+f_{\beta}(k)+\sigma_{\beta}(k),
$$

where $\beta_{o}$ represents the constant term, $f_{\beta}(t)$ represents the slowly varying term with initial value zero, and $\sigma_{\beta}$ represents Gaussian white RNs. For $f_{\beta}(t)$, since its form and polarity are unknown and its values vary slowly, it is better to be omitted in the state equations. For example, if the increasing function is used as the state equation and actually $f_{\beta}(t)$ is decreasing with time, the unmatched model will lead to enlarged estimation errors. Hence, when establishing the registration model, the state equation for any bias can be written as

$$
\beta(k)=\beta_{0}+\sigma_{\beta}(k) .
$$

Comparing with (41), (40) is only used to generate simulation/measurement data in experiments.

For radar OBs, the random term $\sigma_{\beta}$ can be easily classified among random measurement noises (gain of the range bias excepted). However, for ABs, according to (26) and (27), $\sigma_{\beta}$ should be converted as explained in URM. According to (41), when URM is applied for varying $A B$ registration, the form of the state equation remains unchanged; the covariance matrix, however, will be changed.

Substituting (40) into (26) and (27), respectively, we have

$$
\begin{aligned}
& \Delta \theta_{i}^{\prime}(k)=\Delta \theta_{i}-\Delta \phi_{i} \\
& +\frac{\underbrace{}_{i}(k) z_{i}(k)\left[\Delta \psi_{i}+f_{\Delta \psi}(k)+\sigma_{\Delta \psi_{i}}(k)\right]-x_{i}(k) z_{i}(k)\left[\Delta \eta_{i}+f_{\Delta \eta}(k)+\sigma_{\Delta \eta_{i}}(k)\right]}{\approx\left[y_{i}(k) z_{i}(k) \Delta \psi_{i}-x_{i}(k) z_{i}(k) \Delta \eta_{i}\right]+\left[y_{i}(k) z_{i}(k) \sigma_{\Delta \psi_{i}}(k)-x_{i}(k) z_{i}(k) \sigma_{\Delta \eta_{i}}(k)\right]}, \\
& \Delta \varepsilon_{i}^{\prime}(k) \approx \Delta \varepsilon_{i}-\frac{x_{i}(k) \Delta \psi_{i}+y_{i}(k) \Delta \eta_{i}}{\sqrt{x_{i}^{2}(k)+y_{i}^{2}(k)}}-\frac{x_{i}(k) \sigma_{\Delta \psi_{i}}(k)+y_{i}(k) \sigma_{\Delta \eta_{i}}(k)}{\sqrt{x_{i}^{2}(k)+y_{i}^{2}(k)}} .
\end{aligned}
$$

Since RNs of attitude biases are independent, the additional covariance caused by them can be written as

$$
\begin{gathered}
\Delta \sigma_{d\left(\Delta \theta^{\prime}\right)}^{2}=\underbrace{\operatorname{Var}\left\{g_{\Delta \psi_{i}} \sigma_{\Delta \psi_{i}}(k)+g_{\Delta \eta_{i}} \sigma_{\Delta \eta_{i}}(k)\right\}}_{=g_{\Delta \psi_{i}}^{2} \sigma_{\Delta \psi_{i}}^{2}+g_{\Delta \eta_{i}}^{2} \sigma_{\Delta \eta_{i}}^{2}}, \\
g_{\Delta \psi_{i}}=\frac{y_{i}(k) z_{i}(k)}{x_{i}^{2}(k)+y_{i}^{2}(k)}, \quad g_{\Delta \eta_{i}}=\frac{-x_{i}(k) z_{i}(k)}{x_{i}^{2}(k)+y_{i}^{2}(k)},
\end{gathered}
$$

$$
\begin{gathered}
\Delta \sigma_{d\left(\Delta \varepsilon^{\prime}\right)}^{2}=\underbrace{\operatorname{Var}\left\{h_{\Delta \psi_{i}} \sigma_{\Delta \psi_{i}}(k)+h_{\Delta \eta_{i}} \sigma_{\eta_{i}}(k)\right\}}_{=h_{\Delta \psi_{i}}^{2} \sigma_{\Delta \psi_{i}}^{2}+h_{\Delta \eta_{i}}^{2} \sigma_{\Delta \eta_{i}}^{2}}, \\
h_{\Delta \psi_{i}}=\frac{x_{i}(k)}{\sqrt{x_{i}^{2}(k)+y_{i}^{2}(k)}}, \quad h_{\Delta \eta_{i}}=\frac{y_{i}(k)}{\sqrt{x_{i}^{2}(k)+y_{i}^{2}(k)}},
\end{gathered}
$$




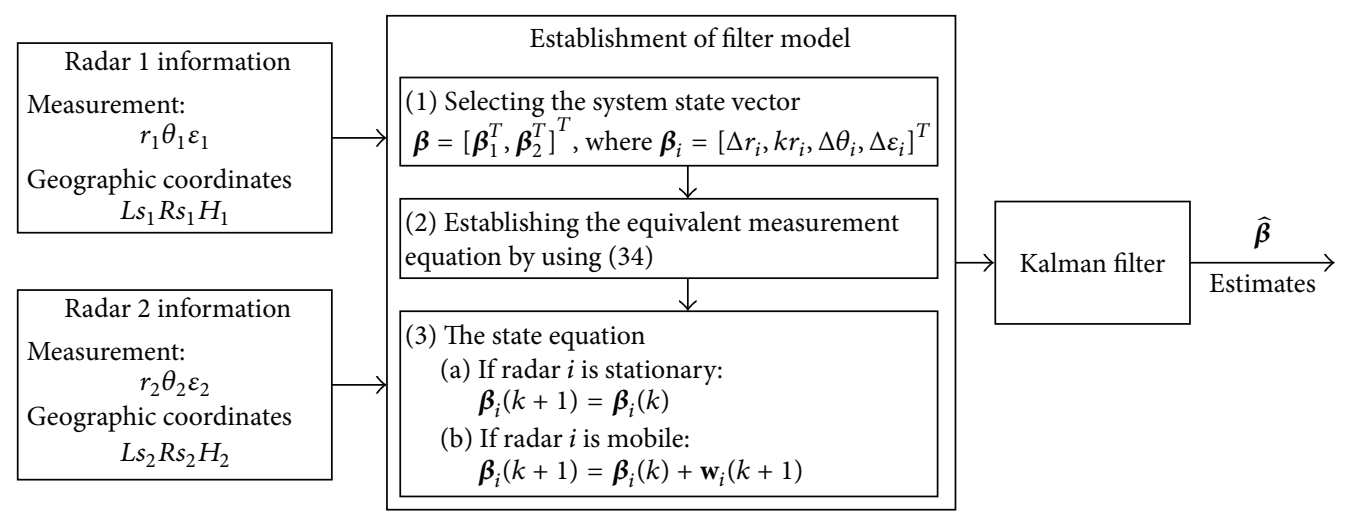

FIGURE 4: The block diagram of URM.

$$
\begin{aligned}
\Delta \sigma_{d\left(\Delta \theta^{\prime}\right) d\left(\Delta \varepsilon^{\prime}\right)}^{2}=E\{ & \left\{g_{\Delta \psi_{i}} \sigma_{\Delta \psi_{i}}(k)+g_{\Delta \eta_{i}} \sigma_{\Delta \eta_{i}}(k)\right] \\
& \left.\times\left[-h_{\Delta \psi_{i}} \sigma_{\Delta \psi_{i}}(k)-h_{\Delta \eta_{i}} \sigma_{\Delta \eta_{i}}(k)\right]\right\} \\
= & -g_{\Delta \psi_{i}} h_{\Delta \psi_{i}} \sigma_{\Delta \psi_{i}}^{2}-g_{\Delta \eta_{i}} h_{\Delta \eta_{i}} \sigma_{\Delta \eta_{i}}^{2} \\
= & \frac{x_{i}(k) y_{i}(k) z_{i}(k)}{\left[x_{i}^{2}(k)+y_{i}^{2}(k)\right]^{3 / 2}}\left(\sigma_{\Delta \eta_{i}}^{2}-\sigma_{\Delta \psi_{i}}^{2}\right) .
\end{aligned}
$$

Then just adding (43) to the corresponding terms of (38), URM can be applied to varying ABs situation.

\section{Simulation Results}

Both URM and OBEM algorithms are tested and compared in a simulated scenario where a common track is generated for two radars which are installed on different ships. As for AAM [1] (the acronym for "all augmented model," where all the OBs and ABs are augmented directly in the state vector), it has been compared with OBEM in [1] and the estimation performance is poorer than OBEM, especially for the elevation biases. Hence, AAM is not compared here.

Assume that ship 1 and ship 2 are moving with the constant velocity model, and the initial geographical coordinates are $\left[40^{\circ}, 116^{\circ}, 10 \mathrm{~m}\right]$ and $\left[40.75^{\circ}, 115.34^{\circ}, 10 \mathrm{~m}\right]$, respectively. Initial states of both ships in their native ENU frame are $[0,10 \mathrm{~m} / \mathrm{s}, 0,10 \mathrm{~m} / \mathrm{s}, 0,0]$. State vector variables are $x$-coordinate (east), $v_{x}$-velocity, $y$-coordinate (north), $v_{y}$-velocity, $z$-coordinate (up), and $v_{z}$-velocity, respectively. Same standard deviations of both ships' process noise are employed in $x, y$, and $z$ coordinates by $0.1 \mathrm{~m} / \mathrm{s}^{2}, 0.1 \mathrm{~m} / \mathrm{s}^{2}$, and $0 \mathrm{~m} / \mathrm{s}^{2}$, respectively. Fusion center locates at the initial position of ship 1 . The constant velocity model is also used for the target. The initial state of the target in fusion center is $[60 \mathrm{~km},-170 \mathrm{~m} / \mathrm{s}, 30 \mathrm{~km}, 30 \mathrm{~m} / \mathrm{s}, 5 \mathrm{~km}, 1 \mathrm{~m} / \mathrm{s}]$. Standard deviations of the process noise in $x, y$, and $z$ coordinates are set to $1 \mathrm{~m} / \mathrm{s}^{2}, 1 \mathrm{~m} / \mathrm{s}^{2}$, and $0.1 \mathrm{~m} / \mathrm{s}^{2}$, respectively.

The geometry of radar and the target is shown in Figure 5. The true systematic OBs of both radars are assumed to be

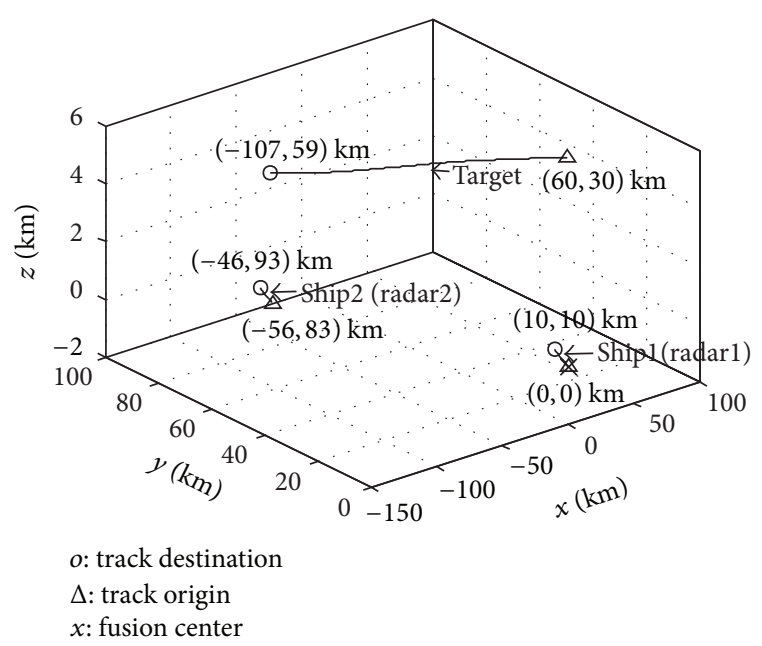

FIGURE 5: The geometry of radar and target.

constant and equal as $\Delta r_{i}=300 \mathrm{~m}, k_{r i}=0.01$, and $\Delta \theta_{i}=$ $\Delta \varepsilon_{i}=2^{\circ}$, respectively.

Standard deviations of the random measurement noises for both radars are $\sigma_{r i}=50 \mathrm{~m}$ and $\sigma_{\theta i}=\sigma_{\varepsilon i}=0.5^{\circ}$, respectively. ABs of both platforms are assumed to be equal with the form of (40), where $\Delta \phi_{i}=\Delta \eta_{i}=\Delta \psi_{i}=1^{\circ}$, and for all $\mathrm{ABs}, f_{\beta}$ is a linear increasing function with $1^{\circ} / h$ slope. The standard deviation of the RNs of all ABs is set equal to $0.1^{\circ}$.

It is assumed that both radars are synchronized with the same sampling intervals $T=5 \mathrm{~s} .200$ scans of the target are simulated and the number of the Monte Carlo runs is set to 100. Figures 6 and 7 contain all the simulation results.

In order to compute the covariance matrix of the state vector $\boldsymbol{\beta}_{\mathrm{URM}}$, it is assumed that the maximum magnitudes are $2^{\circ}$ for each attitude bias of both platforms, and the standard deviation for velocities in $x, y$, and $z$ directions are $[200,200,20] \mathrm{m} / \mathrm{s}$, respectively. Figure 6 depicts the RMSEs of radar bias estimation results. Black solid lines represent the estimation results of OBEM and the red dotted lines represent URM. Thin lines represent radar 1 and thick lines represent radar 2 and green lines represent CRLBs of biases. 


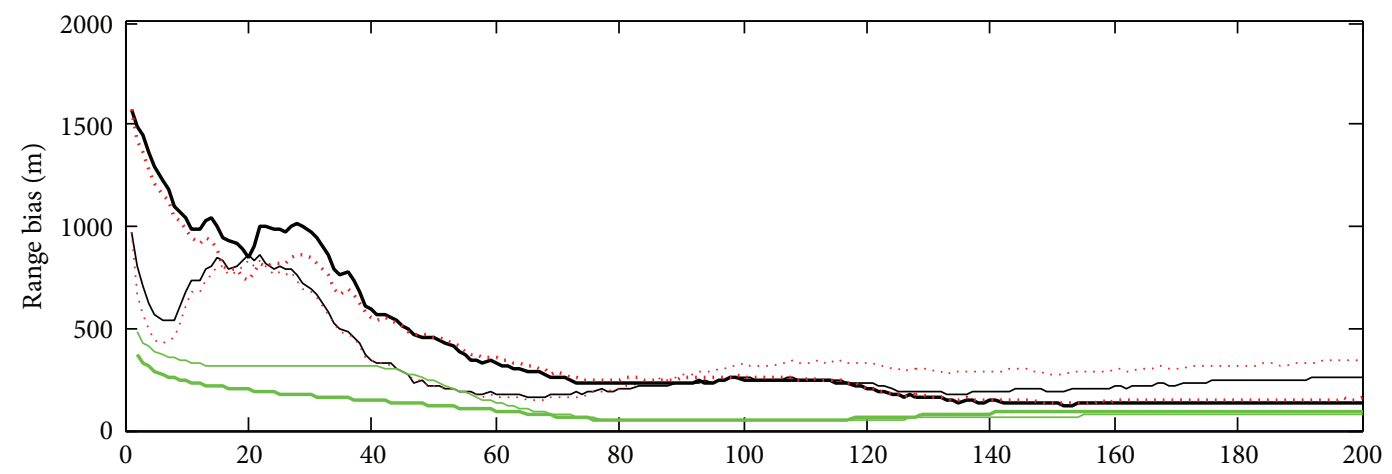

(a) Time step $(T=5 \mathrm{~s})$

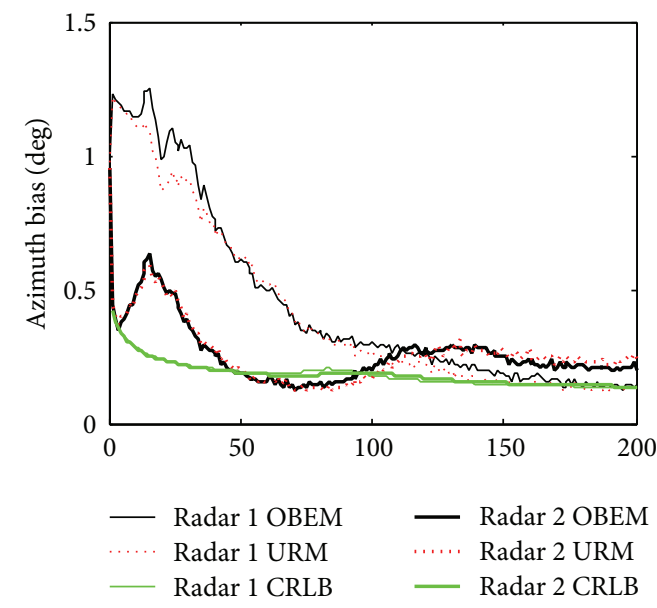

(b) Time step

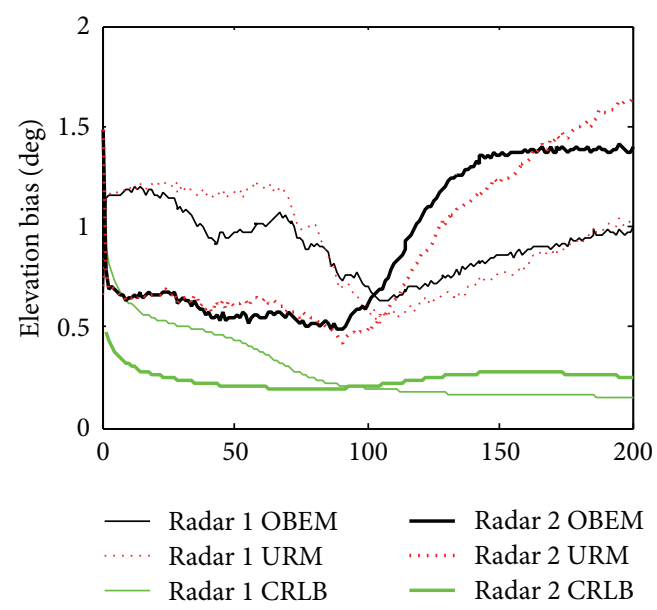

(c) Time step

FIGURE 6: Root mean square errors (RMSEs) of radar gross BEs. (a) Range bias; (b) azimuth bias; (c) elevation bias.

Here, biases denote gross biases which contain radar OBs and equivalent biases caused by ABs. In computation of RMSEs, the true "gross biases" can be obtained by using (12) and (13) with true values. From these figures, we infer that the performance of URM is very close but superior to OBEM as a whole. The RMSE of gross range bias estimation is about $100 \mathrm{~m}$, and the azimuth bias is about $0.1^{\circ}$. However, the gross elevation biases are poor with their RMSEs which are more than $1^{\circ}$.

Usually, for TCs, $z \ll x, y$, according to (12), the pitch and roll biases are minor factors to gross azimuth bias compared with the yaw bias, so they can be omitted and the gross azimuth bias is almost constant. According to (31a), the variance of the gross azimuth bias is small. Hence, the estimation of the gross azimuth bias is good. On the contrary, according to (13) and (32), the variance of the gross elevation bias is big and its values are affected by TCs greatly which are obtained from radar raw measurements and have big errors especially when the target is far away from radar. So, the estimation results for the elevation biases are poor.

Figure 7 is RMSEs of TCs in ECEF frame which are obtained by using radar 1 raw measurements and measurements rectified by BEs, respectively; the thick black line represents RMSEs of the raw measurements; thin black lines represent RMSEs of raw measurements rectified by OBEM; red lines represent URM; and green lines represent the raw measurements rectified by true values of all equivalent biases obtained from (12) and (13). Simulation results show that two methods are almost the same. We infer that lines rectified by URM and OBEM are very close to lines rectified by true bias values in $x$ and $y$ coordinates; however, $z$ coordinates have larger errors. This indicates that URM needs further improvement to improve the elevation bias estimation accuracy.

It should be noted that since the derivations of the model are first-order Taylor series approximations, which corresponds to modeling of small biases applications, when $k_{r i}+\Delta r_{i} / r_{i}$, the sum of angle biases and random measurement noises are on the order of $10^{-2}$; this approximated model works well. In practice (or real world applications), this condition is usually satisfied (e.g., $\Delta \theta_{i}=2^{\circ} \approx 0.03 \mathrm{rad}$ ).

\section{Summary and Conclusion}

For mobile radar, all the attitude biases can be converted into radar measurement errors by three analytical equations (range, azimuth, and elevation angles, resp.). The converted measurement errors have similar influences with radar offset 


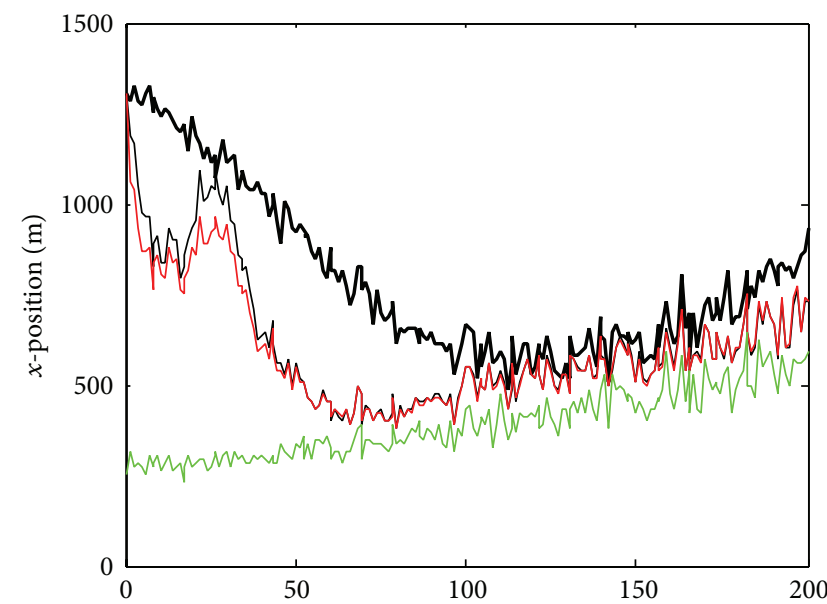

(a) Time step

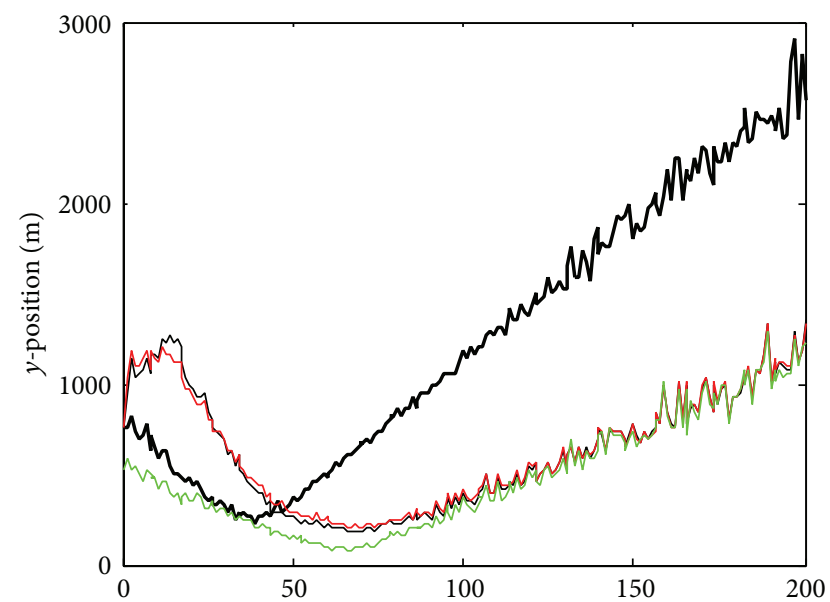

(b) Time step

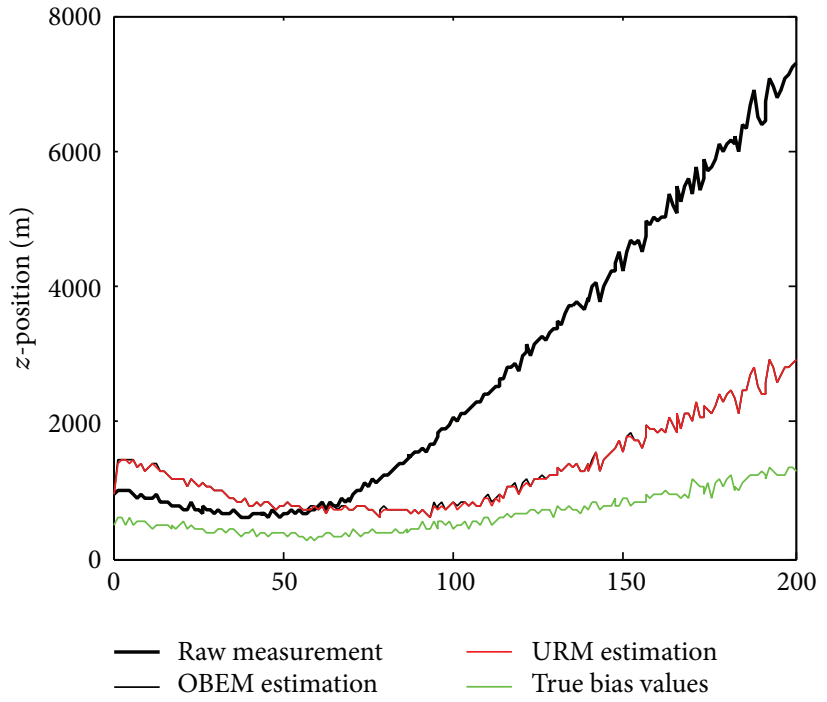

(c) Time step $(T=5 \mathrm{~s})$

FIGURE 7: RMSE of the target location in $x y z$-coordinates after rectifying radar 1 measurements by BEs. (a) $x$-coordinates; (b) $y$-coordinates; (c) $z$-coordinates.

biases on radar measurements. In fact, both kinds of biases are dependent, which was proved in the paper. In other words, we cannot simultaneously estimate offset and attitude biases separately. Based on this, a unified registration model (URM) was proposed whose registration equations only contain radar "offset biases" as state vector. However, these "offset biases" contain the influences of the attitude biases. These equivalent "offset bias" estimates can be used directly to compensate the influences of the attitude and offset biases on radar raw measurements simultaneously. Though URM has the least number of state variables and minor computation burden compared with other mobile radar registration model, URM is guaranteed to be observable. Simulation results show that URM equivalent range and azimuth bias estimates are well and close to the Cramer-Rao low bounds (CRLBs) except that the equivalent elevation bias estimates should be improved further.
Though the paper is about mobile $3 \mathrm{D}$ radar registration, the method and ideas in the paper will be helpful to design all kinds of observation systems on mobile platform.

\section{Appendix}

\section{Cramer-Rao Lower Bound of URM}

The linearized equivalent measurement equation of (34) can be simplified as

$$
\begin{array}{r}
\mathbf{Z}_{\mathrm{URM}}(k)=\mathscr{H}(k) \boldsymbol{\beta}_{\mathrm{URM}}(k)+\mathbb{C}(k) \mathbf{w}(k) \\
(k=1,2, \ldots, N-1) .
\end{array}
$$


In order to get the lowest CRLB, $\boldsymbol{\beta}$ can be viewed as constants since their variations are small. Then the joint probability density function (PDF) can be written as

$$
\begin{aligned}
& p\left(\mathbf{Z}_{k} ; \boldsymbol{\beta}\right) \\
& =\frac{1}{(2 \pi)^{k n / 2} \prod_{i=1}^{k}\left[\left|\mathbb{C}(i) \mathbf{R} \mathbb{C}(i)^{T}\right|^{1 / 2}\right]} \\
& \times \exp \left\{-\frac{1}{2} \sum_{i=1}^{k}\left[\left(\mathbf{Z}_{\mathrm{URM}}(i)-\mathscr{H}(i) \boldsymbol{\beta}\right)^{T}\right.\right. \\
& \times\left(\mathbb{C}(i) \mathbf{R} \mathbb{C}(i)^{T}\right)^{-1} \\
& \left.\left.\times\left(\mathbf{Z}_{\mathrm{URM}}(i)-\mathscr{H}(i) \boldsymbol{\beta}\right)\right]\right\},
\end{aligned}
$$

where $\mathbf{Z}_{k}=\left[\mathbf{Z}_{\mathrm{URM}}(1), \mathbf{Z}_{\mathrm{URM}}(2), \ldots, \mathbf{Z}_{\mathrm{URM}}(k)\right]$, which represents the equivalent measurements up to time $k$.

The Fisher information matrix (FIM) can be given as [16]

$$
\begin{aligned}
\mathbf{J}(m, n) & =E\left\{-\left.\frac{\partial^{2} \ln p\left(\mathbf{Z}_{k} ; \boldsymbol{\beta}\right)}{\partial \boldsymbol{\beta}_{m} \partial \boldsymbol{\beta}_{n}}\right|_{\boldsymbol{\beta}=\boldsymbol{\beta}_{t}}\right\} \\
& =E\left\{\sum_{i=1}^{k}\left[\mathscr{H}(i)^{T}\left(\mathbb{C}(i) \mathbf{R} \mathbb{C}^{T}(i)\right)^{-1} \mathscr{H}(i)\right]\right\},
\end{aligned}
$$

where $\boldsymbol{\beta}_{t}$ denotes the true bias values. However, the expectation in (A.3) with respect to $p(\mathbf{Z}(i) ; \boldsymbol{\beta})$ can hardly be achieved since $\mathscr{H}(i)$ and $\mathbb{C}(i)$ are nonlinear functions of radars' raw measurements $\mathbf{Z}(i)$ radars. The expectation can be approximated by evaluating the mean of Monte-Carlo simulations; that is, (A.3) can be approximated as

$$
\mathbf{J}(m, n) \approx \frac{\sum_{i=1}^{M}\left(\sum_{i=1}^{k}\left[\mathscr{H}(i)^{T}\left(\mathbb{C}(i) \mathbf{R} \mathbb{C}^{T}(i)\right)^{-1} \mathscr{H}(i)\right]\right)}{M}
$$

CRLBs for each bias can be written as

$$
\mathrm{CRLB}_{\boldsymbol{\beta}_{m}}=\mathbf{J}^{-1}(m, m) \text {. }
$$

Equation (A.2) shows that when the biases $\beta$ are constants, we can obtain the lowest CRLB. CRLBs plotted in Figure 6 are calculated under this assumption. As shown in Figure 6, MMSEs of the range and azimuth biases come close to the corresponding CRLBs. As for the elevation biases, their corresponding MMSEs are closer to CRLB curves corresponding to nonconstant equivalent biases; however, the calculated CRLBs correspond to the constant biases assumption, which is the reason why the MMSEs deviate relatively large from calculated CRLBs in Figure 6(c).

\section{Conflict of Interests}

The authors declare that there is no conflict of interests regarding the publication of this paper.

\section{Acknowledgments}

This work was supported in part by the National Natural Science Foundation of China 61032001, 61102165, and 61002006 and Special Foundation Program for Mountain Tai Scholars of China.

\section{References}

[1] G. H. Wang, L. Chen, and S. Y. Jia, "Optimized bias estimation model for 3-D radar considering platform attitude errors," IEEE Aerospace and Electronic Systems Magazine, vol. 27, no. 1, pp. 1924, 2012.

[2] I. F. Progri, Geolocation of RF Signals Principles and Simulations, Springer Science \& Business Media, LLC, New York, NY, USA, 1st edition, 2011.

[3] T. N. Upadhyay, I. F. Progri, J. Lomas, and J. Buckler, "Precision relative navigation for automated rendezvous and docking," in Proceedings of the Annual AAS Guidance and Control, pp. 368379, Breckenridge, Colo, USA, 1999.

[4] Y. Bar-Shalom, "Airborne GMTI radar position bias estimation using static-rotator targets of opportunity," IEEE Transactions on Aerospace and Electronic Systems, vol. 37, no. 2, pp. 695-699, 2001.

[5] S. M. Herman and A. B. Poore, "Nonlinear least-squares estimation for sensor and navigation biases," in Signal and Data Processing of Small Targets, vol. 6263 of Proceedings of SPIE, April 2006.

[6] B. D. Kragel, S. S. Danford, M. Herman, and A. B. Poore, "Bias estimation using targets of opportunity," in Signal and Data Processing of Small Targets, vol. 6699 of Proceedings of SPIE, 2007.

[7] L. Chen, G. H. Wang, S. Y. Jia, and I. Progri, "Attitude bias conversion model for mobile radar error registration," Journal of Navigation, vol. 65, no. 4, pp. 651-670, 2012.

[8] G. H. Wang, L. Chen, S. Y. Jia, and I. Progri, "Optimized bias estimation model for mobile radar error registration," The Journal of Navigation, vol. 66, no. 2, pp. 227-248, 2013.

[9] R. Hermann and A. J. Krener, "Nonlinear controllability and observability," IEEE Transactions on Automatic Control, vol. 22, no. 5, pp. 728-740, 1977.

[10] A. D. King, "Inertial navigation-past, present, and future," in Proceedings of the IEE Colloquium on Airborne Navigation Systems Workshop, pp. 3/1-3/9, London, UK, 1997.

[11] R. E. Helmick and T. R. Rice, "Removal of alignment error in an integrated system of two 3-D sensors," IEEE Transactions on Aerospace and Electronic Systems, vol. 29, no. 4, pp. 1333-1343, 1993.

[12] U. I. Bhatti and W. Y. Ochieng, "Failure modes and models for integrated GPS/INS systems," The Journal of Navigation, vol. 60, no. 2, pp. 327-348, 2007.

[13] W.-K. Tseng and H.-S. Lee, "The vector function for distance travelled in great circle navigation," The Journal of Navigation, vol. 60, no. 1, pp. 158-170, 2007.

[14] Y. Zhou, H. Leung, and M. Blanchette, "Sensor alignment with earth-centered earth-fixed (ECEF) coordinate system," IEEE Transactions on Aerospace and Electronic Systems, vol. 35, no. 2, pp. 410-418, 1999.

[15] Y. Y. Qin, H. Y. Zhang, and S. H. Wang, Kalman Filter and Integrated Navigation Theory, Northwestern Polytechnic University Press, Xi'an, China, 1998 (Chinese).

[16] S. M. Kay, Fundamental of Statistical Signal Processing: Estimation Theory, chapter 3, Prentice-Hall, Englewood Cliffs, NJ, USA, 1993. 

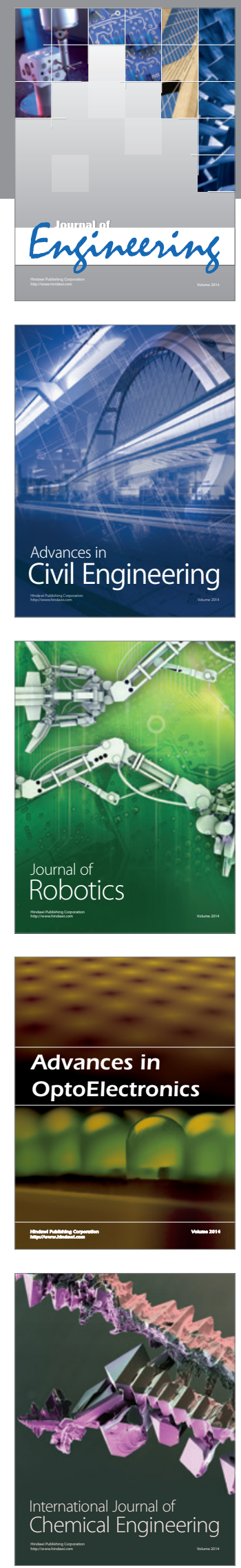

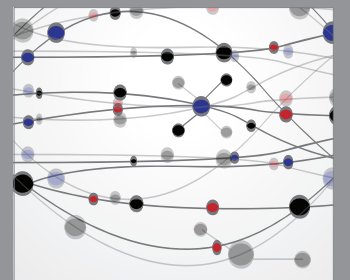

The Scientific World Journal
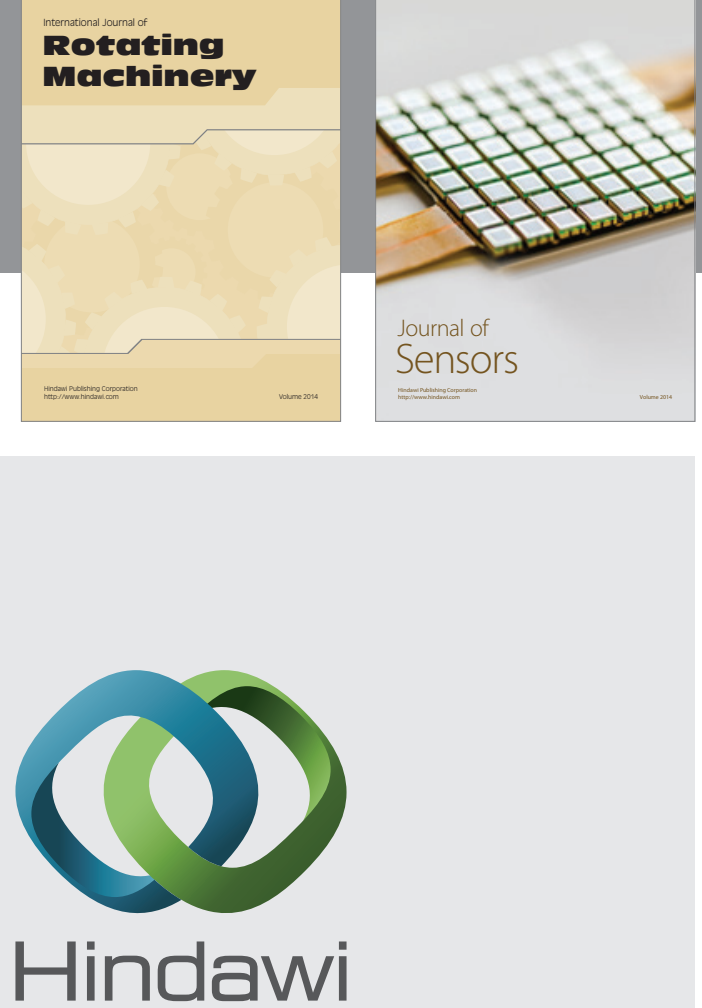

Submit your manuscripts at http://www.hindawi.com
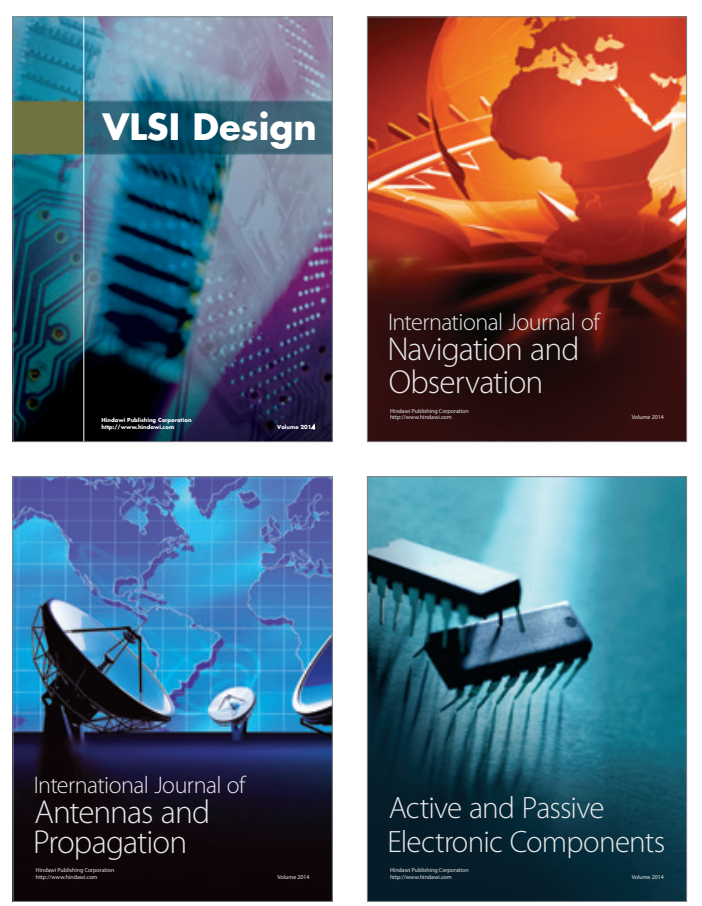
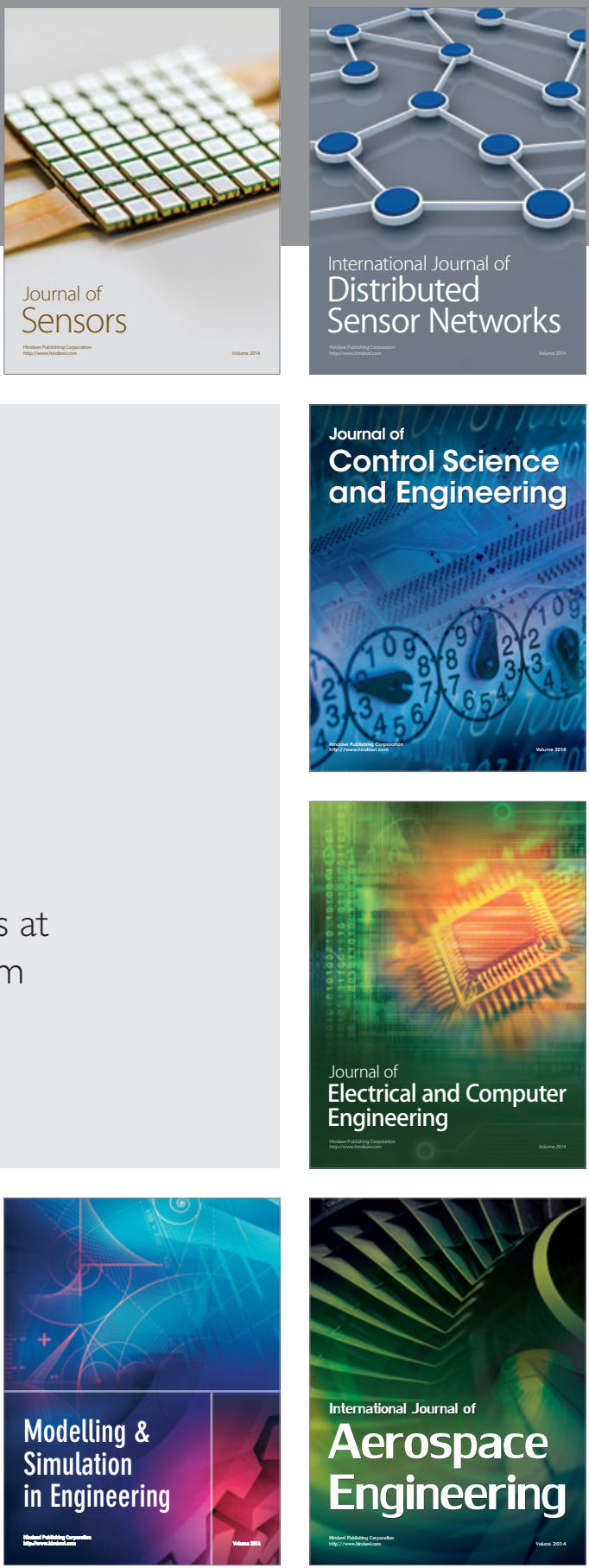

Journal of

Control Science

and Engineering
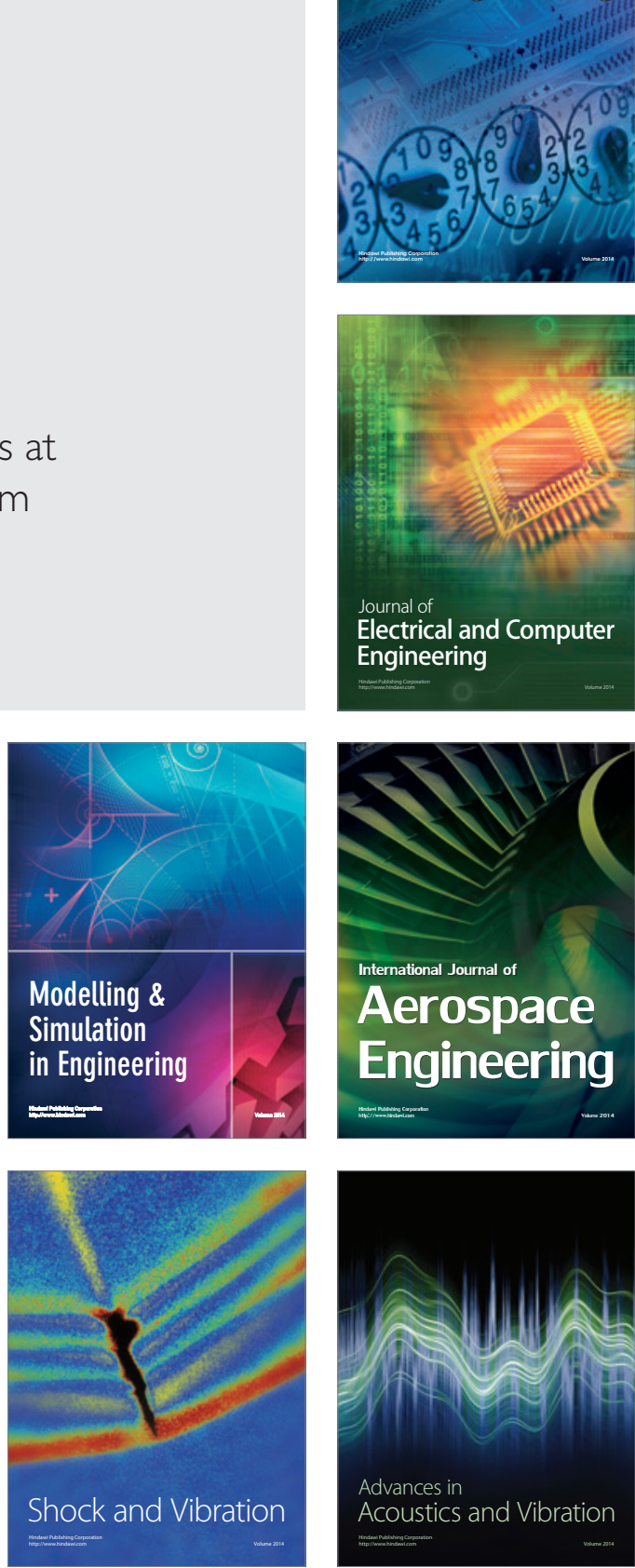